\title{
HIPERTENSÃO MASCARADA: QUAL O VERDADEIRO RISCO CARDIOVASCULAR?
}

\author{
MASKED HYPERTENSION: WHAT IS THE REAL CARDIOVASCULAR RISK?
}

Sergio Emanuel Kaiser ${ }^{1}$, Ronaldo Gismondi²

\section{RESUMO}

Entende-se como hipertensão mascarada (HM) a existência de níveis pressóricos aumentados fora do consultório em pessoas supostamente normotensas e não tratadas. A hipertensos medicados, aplica-se a denominação de "hipertensão mascarada não controlada" (HMNC). Estas condições expõem expressivo contingente de indivíduos a um risco não identificado para eventos cardiovasculares. O presente trabalho teve como objetivo realizar uma revisão sistemática da literatura a fim de identificar os principais estudos de associação entre HM, HMNC e o risco de eventos cardiovasculares. De um total de 566 estudos, 19 foram incluídos na revisão. Dentre estes, apenas 4 não documentaram associação entre HM/HMNC e maior risco cardiovascular. Um estudo observou apenas associação com risco de infarto agudo do miocárdio (IAM) e outro apenas com o risco de acidente cerebrovascular (AVC). Os demais 13 estudos mostraram relação entre presença de HM e/ou HMNC e maior risco de eventos cardiovasculares como AVC, IAM e/ou morte. Em conclusão, existe associação entre a presença de hipertensão mascarada e o aumento no risco de eventos cardiovasculares. Alguns fenótipos especialmente vulneráveis e possíveis estratégias diagnósticas são também objeto de discussão.

Descritores: Hipertensão; Monitorização Ambulatorial da Pressão Arterial; Hipertensão Mascarada.

\section{ABSTRACT}

Masked hypertension $(\mathrm{MH})$ is defined as a normal ambulatory blood pressure, though elevated in the outpatient setting, in supposedly normotensive patients. For hypertensive patients, the term "uncontrolled masked hypertension" (MUCH) applies. Previous data suggest that subjects who present either MH or MUCH may be exposed to higher cardiovascular risk. The authors sought to carry out a systematic review of the literature regarding the association between $\mathrm{MH}, \mathrm{MUCH}$ and risk of cardiovascular events. Among 566 studies retrieved, 19 were included in the review. Only 4 studies did not document an association between $\mathrm{MH} / \mathrm{MUCH}$ and risk of cardiovascular events. One study found an association only with the risk of acute myocardial infarction (AMI) and another with the risk of cerebrovascular events. The remaining 13 studies revealed a relationship between the presence of $\mathrm{MH} / \mathrm{MUCH}$ and a higher risk of cardiovascular events such as stroke, AMI and/or death. In conclusion, there is an association between the presence of $\mathrm{MH} / \mathrm{MUCH}$ and an increased risk of cardiovascular events. Some especially vulnerable phenotypes as well as possible diagnostic strategies are also discussed.

Keywords: Hypertension; Masked Hypertension; Ambulatory Blood Pressure Monitoring.

\section{INTRODUÇÃO}

A hipertensão arterial desponta como o principal fator de risco envolvido na mortalidade cardiovascular em nosso país ${ }^{1}$ e, não obstante o aumento expressivo na taxa de conhecimento e tratamento desta condição ao longo dos anos, a proporção de indivíduos com a pressão arterial controlada está estacionada na faixa de 10 a 35\% dos casos, a depender da região geográfica analisada. ${ }^{2}$ Estes dados, por si só decepcionantes, refletem uma ênfase na medida ambulatorial da pressão arterial, mas a realidade é ainda mais preocupante ao ajuntar-se, ao precário controle dos demais fatores de risco cardiovascular (honrosa exceção para o tabagismo) a insuficiente redução a níveis seguros das cifras tensionais nas 24 horas, particularmente durante o sono. O descenso inadequado e a elevação da pressão arterial (PA) durante o sono associam-se respectivamente a aumento de 12-23\% e 57-89\% no risco de eventos cardiovasculares e cerebrovasculares. ${ }^{3}$ Uma apresentação cujo valor prognóstico vem ganhando mais reconhecimento, expressa-se através do fenótipo conhecido como "hipertensão mascarada".

1. Disciplina de Fisiopatologia Clínica e Experimental (Clinex), Universidade do Estado do Rio de Janeiro (UERJ), Rio de Janeiro, RJ, Brasil.

2. Departamento de Medicina Clínica (MMC), Universidade Federal Fluminense (UFF), Rio de Janeiro, RJ, Brasil. 
Os termos "hipertensão mascarada" ou "hipertensão ambulatorial não detectada" - este último abandonado - foram cunhados pela primeira vez por Thomas Pickering em 2002, ${ }^{4}$ a partir da identificação, pelo mesmo grupo, do protagonismo deste fenômeno no aumento da prevalência de hipertrofia ventricular esquerda e de placas ateroscleróticas carotídeas em pacientes hipertensos. ${ }^{5}$ Antes de discuti-la, porém, cabe definir mais precisamente a terminologia: Entende-se como hipertensão mascarada (HM) a existência de níveis pressóricos aumentados fora do consultório em pessoas supostamente normotensas e não tratadas. Portanto, esta definição aplicar-se-á a normotensos sem tratamento com pressão arterial inferior a 140/90 mmHg em ambulatório e valores médios durante a vigília iguais ou acima de 135/85 mm tanto à monitorização ambulatorial da pressão arterial (MAPA) como à medida da pressão arterial fora do ambulatório. Nestes indivíduos, a média das cifras durante o período de sono igual ou maior que 120/70 mmHg ou a média de 24 horas acima de 130/80 mmHg, identificadas por meio de MAPA, também diagnosticam HM. ${ }^{6} \mathrm{~A}$ hipertensos medicados, com PA aparentemente controlada em ambulatório, mas não fora dele, aplica-se a denominação de "hipertensão mascarada não controlada" (HMNC). A importância desta distinção prende-se à maior chance de expor um expressivo contingente de indivíduos aparentemente normotensos a um risco cardiovascular não identificado: Através de MAPA ou automedida da pressão arterial (AMPA) estima-se, em pacientes com PA normal em ambulatório, uma prevalência entre7,5 a 28\% para $\mathrm{HM}$ e de 29 a $43 \%$ para HMNC. ${ }^{7-12}$

\section{OBJETIVO}

Este trabalho teve por objetivo realizar uma revisão sistemática da literatura a fim de identificar os principais estudos de associação entre HM, HMNC e risco de eventos cardiovasculares.

\section{MÉTODOS}

Foi realizada uma revisão sistemática em observância aos critérios estabelecidos pelo Preferred Reporting Items For Systematic Reviews and Meta-Analyses (PRISMA).

\section{Critérios de Elegibilidade}

Estudos longitudinais em pacientes com $\geq 18$ anos de idade sobre prognóstico cardiovascular em pacientes com HM e/ou HMNC. Os desfechos de interesse foram acidente vascular cerebral, infarto agudo do miocárdio e mortalidade (cardiovascular e/ou geral). Foram excluídos estudos com pacientes em hemodiálise.

\section{BASE BIBLIOGRÁFICA}

Para pesquisa dos artigos empregou-se a base de dados PubMed, com artigos em português ou inglês publicados até janeiro de 2020. A estratégia de busca selecionou as palavras-chave "masked hypertension" e "cardiovascular events". As palavras "risk" e "prognosis" foram utilizadas como alternativa a "events". As buscas foram complementadas com a análise das referências de todos os artigos selecionados para leitura na íntegra.

Os dois autores realizaram a busca de modo independente e os cruzaram-se os resultados a fim de selecionar as publicações incluídas na revisão. Em caso de discordância, a decisão deu-se através de consenso dos autores.

\section{RESULTADOS}

Dentre 566 estudos identificados, 20 foram selecionados para leitura na íntegra. Um artigo foi excluído por avaliar pacientes em diálise, restando 19 estudos para análise. (Tabela 1) Dos 19 estudos, 4 não documentaram associação entre HM/HMNC e risco de eventos cardiovasculares. Um estudo observou apenas associação com risco de infarto agudo do miocárdio (IAM) e outro apenas com o risco de acidente cerebrovascular (AVC). Os demais 13 estudos mostraram relação entre presença de $\mathrm{HM}$ e/ou HMNC e maior risco de eventos cardiovasculares como AVC, IAM e/ou morte. (Tabela 1)

\section{DISCUSSÃO}

$\mathrm{Na}$ presente revisão sistemática da literatura, a maior parte dos estudos revelou associação entre a presença de hipertensão mascarada e o aumento no risco de eventos cardiovasculares. O papel prognóstico da MAPA e da monitorização residencial da PA é bem sedimentado na literatura: Estas formas de aferição da PA demonstram melhor correlação com risco cardiovascular quando comparados à medida da pressão em consultório. ${ }^{2,11,12}$ Não surpreende, portanto, a associação entre níveis tensionais aumentados nas 24 horas e maior risco cardiovascular mesmo perante uma PA normal aferida em ambulatório.

A magnitude da associação entre HM e risco de eventos cardiovasculares nos dois maiores estudos situa-se em torno de 2,0 a 2,7 vezes. ${ }^{13,14}$ Apenas um estudo mostrou associação extremamente alta, mas a população estudada era de renais crônicos em tratamento conservador, já sabidamente de maior risco em relação àqueles com função renal normal. ${ }^{15}$ Em recente metanálise, com perfil de inclusão de estudos semelhante a esta revisão, ${ }^{7}$ o risco relativo para eventos cardiovasculares maiores (MACE) foi de 2,09 (IC 95\% 1,80-2,44).

Discute-se a existência de diferenças entre o efeito "mascarado" em indivíduos não hipertensos (HM) e em hipertensos que não obstante o tratamento, exibem fenômeno do mascaramento (HMNC). Nos estudos incluídos nesta revisão sistemática e na meta-análise recente já citada, a magnitude do risco relativo foi semelhante entre HM e HMNC. Tal resultado corrobora o conceito de que o aumento na média das cifras tensionais é um dos principais determinantes do risco de eventos cardiovasculares, independente do uso ou não de fármacos hipotensores.

Na presente revisão, os estudos com menor seguimento duraram cerca de 3 anos e os maiores entre 10 e 15 anos. Este dado aponta para a existência de um risco atribuído à HM em prazo não muito longo, e, portanto, para a importância de não negligenciar a sua presença.

Longe, portanto, de representar um preciosismo diagnóstico, a HM pode evoluir para hipertensão sustentada em até metade dos casos em cinco anos ${ }^{16}$ e acelerar o desenvolvimento de lesões em órgãos-alvo quando não reconhecida precocemente. ${ }^{17,18}$ A HM é mais prevalente em indivíduos mais jovens quando comparados a idosos ${ }^{19}$ e alguns fenótipos especialmente predispostos a apresentar HM poderão merecer, portanto, uma estratégia diagnóstica diferenciada: enquadram-se nesta categoria portadores de diabetes mellitus, ${ }^{20,21}$ apneia obstrutiva do sono, ${ }^{22}$ doença renal crônica, ${ }^{23}$ 
Tabela 1. Estudos avaliando risco cardiovascular em HM. MACE: Eventos cardiovasculares maiores. IC: Insuficiência cardíaca. FA: Fibrilação atrial AVC: Acidente cerebrovascular. MRPA: Monitorização residencial da pressão arterial.

\begin{tabular}{|c|c|c|c|}
\hline Referência & $\begin{array}{l}\text { Participantes } \\
\text { (Total/HM) }\end{array}$ & $\begin{array}{l}\text { Seguimento } \\
\text { (anos) }\end{array}$ & Risco relacionado a eventos cardiovasculares \\
\hline Bobrie, $2004^{14}$ & $4939 / 462^{* * * *}$ & 3,2 & Razão de chances de 2,06 $(1,22-3,47)$ para MACE \\
\hline Fagard, $2005^{31}$ & $391 / 31$ & 10,9 & Sem associação estatisticamente significativa com MACE \\
\hline Pierdomenico, $2005^{32}$ & $466 / 126$ & 5 & Razão de chances de $2,28(1,10-4,70)$ para MACE \\
\hline Ohkubo, $2005^{33}$ & $1332 / 221$ & 10 & Razão de chances de $2,13(1,38-3,29)$ para MACE \\
\hline Ben-Dov, $2008^{34}$ & $2285 / 268$ & 7,7 & Razão de chances de $1,88(1,08-3,27)$ para MACE \\
\hline Hansen, $2006^{35}$ & $1700 / 211$ & 9,5 & Sem associação estatisticamente significativa com MACE \\
\hline Mancia, $2006 \S^{36}$ & $1863 / 184$ & 12,3 & Razão de chances de 5,57 para mortalidade cardiovascular \\
\hline Pierdomenico, $2008^{37}$ & $591 / 120^{*}$ & 6,6 & Razão de chances de 2,65 $(1,18-5,98)$ para MACE \\
\hline Kushiro, $2008 \S^{38}$ & $2896 / 566$ & 3,5 & $\begin{array}{l}\text { HM não aumentou risco de MACE comparado a hipertensos } \\
\text { tratados e controlados }\end{array}$ \\
\hline Hermida, $2012^{39}$ & $3344 / 354$ & 5,6 & Razão de chances de 2,48 $(1,02-5,99)$ para MACE \\
\hline Hanninen, $2012^{40}$ & 2046/188 & 7,5 & $\begin{array}{l}\text { Razão de chances de } 2,29(1,43-3,68) \text { para MACE, mas análise } \\
\text { multivariada para idade e sexo, removeu significância estatística }\end{array}$ \\
\hline Minutolo, $2014^{41}$ & $498 / 71^{* *}$ & 5,2 & Razão de chances de 3,17 $(1,50-6,69)$ para MACE \\
\hline Tientcheu, $2015 \S^{18}$ & $3027 / 582$ & 9 & $\begin{array}{l}\text { Razão de chances de } 2,03(1,36-3,03) \text { para desfecho } \\
\text { combinado de MACE, IC e FA }\end{array}$ \\
\hline Booth, $2016^{42}$ & $738 / 385^{* * *}$ & 8,2 & Razão de chances de 2,49 (1,26-4,93) para MACE \\
\hline Wang, $2017^{15}$ & $588 / 121^{* *}$ & 2,9 & Razão de chances de 8,66 $(1,09-68,79)$ para MACE \\
\hline Pannarale, $2017^{43}$ & $204 / 62 *$ & 15,6 & Sem houve associação estatisticamente significativa com MACE \\
\hline Pierdomenico, $2017^{44}$ & $1191 / 142^{* * * *}$ & 9,1 & Razão de chances de 1,60 $(1,12-2,29)$ para MACE \\
\hline Tocci, $2018^{45}$ & $2209 / 149$ & 10 & $\begin{array}{l}\text { Razão de chances de } 4,11(1,69-10,01) \text { apenas para IAM, mas } \\
\text { não para AVC ou mortalidade }\end{array}$ \\
\hline Fujiwara, $2018 \S^{13}$ & $4261 / 810$ & 3,9 & $\begin{array}{l}\text { Razão de chances de } 2,77(1,20-6,37) \text { para AVC, mas não } \\
\text { significativo para doença coronariana. }\end{array}$ \\
\hline
\end{tabular}

obesidade visceral - especialmente quando acompanhada de pressão arterial limítrofe ${ }^{24}$ - e pré-hipertensos diagnosticados em ambulatório. ${ }^{25,26}$ Por sua vez, a HMNC pode simplesmente refletir um estado intermediário, de controle incompleto da PA motivado pela falsa sensação de segurança oferecida pelo achado de normotensão num momento em que se manifesta o efeito mais intenso de drogas hipotensoras, nem sempre eficazes num ciclo de 24 horas.

\section{QUEM DEVE SER RASTREADO E DE QUE FORMA?}

Diante da importância prognóstica da HM e HMNC, é imperativo definir uma estratégia de identificação deste grupo especialmente vulnerável: Quem deve ser investigado e qual a ferramenta mais adequada? Num hipotético cenário ideal, todo adulto normotenso deveria ao menos verificar sua pressão arterial com aparelho digital semiautomático fora do ambiente ambulatorial. No entanto, a AMPA requer, para um diagnóstico correto, o cumprimento de uma série de instruções básicas, algum tempo para treinar os pacientes, disponibilidade de pessoal para esta finalidade e, por fim, algum recurso financeiro para aquisição de uma boa e validada ferramenta de aferição. Como exemplo da inexequibilidade de uma triagem universal, um estudo norte-americano estimou em cerca de 120 milhões o número de cidadãos com indicação de submeter-se a algum método não ambulatorial a fim de detectar o maior contingente possível de portadores de hipertensão mascarada. ${ }^{27}$ As sétimas diretrizes brasileiras de hipertensão arterial estimam em 13\% a prevalência de $\mathrm{HM}$, reconhecem o maior risco cardiovascular atribuído à sua identificação e a maior prevalência desta condição em pacientes com níveis tensionais limítrofes em ambulatório, mas não recomendam uma estratégia diagnóstica específica para a identificação de portadores desta condição. ${ }^{2}$ As mais recentes diretrizes europeias recomendam especial atenção a portadores de pressão arterial limítrofe em ambulatório, àqueles com lesões em órgãos-alvo relacionadas à hipertensão identificadas durante a consulta, e aos possuidores de alto risco de eventos cardiovasculares. ${ }^{11}$ Em afro-americanos, a identificação de risco para eventos cardiovasculares em dez anos igual ou superior a 7,5\% associou-se a uma prevalência de $\mathrm{HM}$ em $63 \%$ dos casos. $^{6}$ As diretrizes norte-americanas de 2017 recomendam a pesquisa de $\mathrm{HM}$ em pessoas cujas cifras tensionais sistólicas e diastólicas em ambulatório se mantenham respectivamente entre 120-129 e 85-89 mmHg após três meses de insistência para induzir mudanças no estilo de vida. ${ }^{12}$ Estas recomendações não contemplam indicadores de risco cardiovascular ou pesquisa de lesões em órgãos-alvo e basearam-se num estudo onde a prevalência de 7,5\% de HM em indivíduos com PA em níveis ótimos contrastava com a presença desta condição em $29 \%$ dos pré-hipertensos. ${ }^{9}$ Até o presente momento, vale ressaltar, não há publicações que permitam comprovar a eficácia da intervenção farmacológica em portadores de HM para prevenir desfechos clínicos, embora particularmente na presença de lesões em órgãos-alvo o tratamento farmacológico soe como alternativa de puro bom 
senso. ${ }^{11,19}$ A HMNC, por outro lado, poderia refletir simplesmente adesão inadequada ao regime terapêutico proposto, mas dados recentemente publicados, valendo-se de dosagem de concentrações urinárias de fármacos em pacientes com e sem HMNC não corroboraram esta impressão, ainda que alguns casos pudessem de fato resultar de adesão imprópria. ${ }^{28}$ Ao contrário, um grande registro espanhol, ao documentar a presença de HMNC em 30\% dos hipertensos tratados, encontrou importante associação com comorbidades tais como diabetes mellitus e doença renal crônica. ${ }^{10} \mathrm{~A}$ medida da PA fora do ambiente ambulatorial padece da limitação imposta pela impossibilidade de obter registros durante o período de sono, quando é possível detectar padrões "non-dipper" ou "dipper reverso", reconhecidamente associados a pior prognóstico. ${ }^{2}$ Talvez por conta desta limitação, num estudo onde os dois métodos foram testados em 333 pessoas normotensas em consultório, o achado de HM em $11 \%$ deles através de AMPA foi inferior aos 25,8\% diagnosticados com esta condição pela MAPA. ${ }^{29}$ Contudo, não há evidências conclusivas sobre a superioridade de um ou outro método para predizer mortalidade ou eventos cardiovasculares. ${ }^{30}$ As sétimas diretrizes brasileiras de hipertensão arterial recomendam um ou outro método "dependendo da indicação, disponibilidade, facilidade, custo de utilização e, se for o caso, preferência do

\section{REFERÊNCIAS}

1. Number of deaths by risk factor [Internet]. Our World in Data. [citado 2 de fevereiro de 2020]. Disponível em: https://ourworldindata.org/grapher/ number-of-deaths-by-risk-factor

2. Malachias MVB. 7th Brazilian Guideline of Arterial Hypertension: Presentation. Arq Bras Cardiol. 2016;107:15-19.

3. Salles GF, Reboldi G, Fagard RH,et al. Pierdomenico Sante D., Verdecchia Paolo, et al. Prognostic Effect of the Nocturnal Blood Pressure Fall in Hypertensive Patients. Hypertension. 2016;67:693-700.

4. Pickering TG, Davidson K, Gerin W, Schwartz JE. Masked hypertension. Hypertension. 2002;40(6):795-796.

5. Liu JE, Roman MJ, Pini R, Schwartz JE, Pickering TG, Devereux RB. Cardiac and Arterial Target Organ Damage in Adults with Elevated Ambulatory and Normal Office Blood Pressure. Ann Intern Med. 1999;131:564.

6. Anstey DE, Booth JN 3rd, Abdalla M, et al. Predicted Atherosclerotic Cardiovascular Disease Risk and Masked Hypertension Among Blacks in the Jackson Heart Study. Circ Cardiovasc Qual Outcomes. 2017;10(7):e003421.

7. Thakkar HV, Pope A, Anpalahan M. Masked Hypertension: A Systematic Review. Heart Lung Circ. 2020;29:102-11.

8. Alwan H, Pruijm M, Ponte B, et al. Epidemiology of Masked and WhiteCoat Hypertension: The Family-Based SKIPOGH Study. PLOS ONE. 2014;9:e92522.

9. Brguljan-Hitij J, Thijs L, Li Y, et al. Risk Stratification by Ambulatory Blood Pressure Monitoring Across JNC Classes of Conventional Blood Pressure. Am J Hypertens. 2014;27:956-65.

10. Banegas JR, Ruilope LM, de la Sierra A, de la Cruz JJ, et al. High prevalence of masked uncontrolled hypertension in people with treated hypertension. Eur Heart J. 2014;35:3304-12.

11. Williams B, Mancia G, Spiering W, et al. 2018 ESC/ESH Guidelines for the management of arterial hypertensionThe Task Force for the management of arterial hypertension of the European Society of Cardiology (ESC) and the European Society of Hypertension (ESH). Eur Heart J. 2018;39:3021-104.

12. Whelton PK, Carey RM, Aronow WS, et al. 2017 ACC/AHA/AAPA/ABC/ ACPM/AGS/APhA/ASH/ASPC/NMA/PCNA Guideline for the Prevention, Detection, Evaluation, and Management of High Blood Pressure in Adults: A Report of the American College of Cardiology/American Heart Association Task Force on Clinical Practice Guidelines. Hypertension. 2018;71(6):e13-e115

13. Fujiwara T, Yano Y, Hoshide S, Kanegae H, Kario K. Association of paciente." ${ }^{2}$ As diretrizes norte-americanas de 2017 não fazem distinção $^{12}$ e as diretrizes europeias de 2018 recomendam a MAPA diante da suspeita de hipertensão noturna, mais frequente em apneia obstrutiva do sono, doença renal crônica, diabetes, hipertensão endócrina ou disfunção autonômica. ${ }^{11}$

\section{PONTOS-CHAVE}

- Hipertensão mascarada é definida por níveis tensionais elevados fora de ambiente ambulatorial em indivíduos aparentemente normotensos.

- A hipertensão mascarada associa-se a risco de eventos cardiovasculares duas a três vezes maior quando comparada a normotensão.

- A magnitude do aumento no risco cardiovascular parece ser a mesma para hipertensão mascarada e em hipertensos com fenômeno de "mascaramento".

- Certos fenótipos são mais propensos a exibir hipertensão mascarada, como renais crônicos, diabéticos, portadores de apneia obstrutiva do sono e obesidade visceral.

- A MAPA é o método ideal para diagnóstico de hipertensão mascarada, mas a medida da pressão arterial fora de ambiente ambulatorial pelos próprios pacientes constitui ferramenta útil na detecção de expressivo percentual de casos.

Cardiovascular Outcomes With Masked Hypertension Defined by Home Blood Pressure Monitoring in a Japanese General Practice Population. JAMA Cardiol. 2018;3:583-90.

14. Bobrie G, Chatellier G, Genes N, Clerson P, Vaur L, Vaisse B, et al. Cardiovascular Prognosis of Masked Hypertension Detected by Blood Pressure Self-measurement in Elderly Treated Hypertensive Patients. JAMA. 2004;291:1342-9.

15. Wang C, Zhang J, Li Y, et al. Masked hypertension, rather than white-coat hypertension, has a prognostic role in patients with non-dialysis chronic kidney disease. Int J Cardiol. 2017;230:33-9.

16. Trudel X, Milot A, Brisson C. Persistence and Progression of Masked Hypertension: A 5-Year Prospective Study. Int J Hypertens. 2013;2013:1-7.

17. Hänninen $M-R$, Niiranen $T$, Puukka $P$, Kesäniemi $Y$, Kähönen $M$, Jula A. Target organ damage and masked hypertension in the general population: the Finn-Home study. J Hypertens. 2013;31:1136-43.

18. Tientcheu D, Ayers C, Das SR, et al. Target Organ Complications and Cardiovascular Events Associated with Masked Hypertension and White Coat Hypertension: Analysis from the Dallas Heart Study. J Am Coll Cardiol. 2015;66:2159-69.

19. Cuspidi C, Sala C, Tadic M, Rescaldani M, Grassi G, Mancia G. Untreated Masked Hypertension and Subclinical Cardiac Damage: A Systematic Review and Meta-analysis. Am J Hypertens. 2015;28:806-13.

20. Franklin SS, Thijs L, Li Y, et al. Masked Hypertension in Diabetes Mellitus: Treatment Implications for Clinical Practice. Hypertension. 2013;61:964-71.

21. Zhou J, Liu C, Shan P, Zhou Y, Xu E, Ji Y. Prevalence and distinguishing features of masked hypertension in type 2 diabetic patients. J Diabetes Complications. 2013;27:82-6.

22. Drager LF, Diegues-Silva L, Diniz PM, et al. Obstructive Sleep Apnea, Masked Hypertension, and Arterial Stiffness in Men. Am J Hypertens. 2010;233:249-54.

23. Parati G, Ochoa JE, Bilo G, et al. Hypertension in Chronic Kidney Disease Part 2. Hypertension. 2016;67:1102-10.

24. Unsal S, Ozkara A, Albayrak T, Ozturk Y, Beysel S, Kucukler FK. Evaluation of prehypertension and masked hypertension rate among clinically normotensive patients. Clin Exp Hypertens. 2016;38:218-24.

25. Barroso WKS, Feitosa ADM, Barbosa ECD, et al. Prevalence of Masked and White-Coat Hypertension in Pre-Hypertensive and Stage 1 Hypertensive patients with the use of TeleMRPA. Arq Bras Cardiol. 2019;1135:970-5. 
26. Anstey DE, Moise N, Kronish I, Abdalla M. Masked Hypertension: Whom and How to Screen? Curr Hypertens Rep. 2019;21:26.

27. Booth JN, Muntner P, Diaz KM, et al. Evaluation of Criteria to Detect Masked Hypertension. J Clin Hypertens. 2016;18:1086-94.

28. Siddiqui M, Judd EK, Dudenbostel T, et al. Masked Uncontrolled Hypertension Is Not Attributable to Medication Nonadherence. Hypertension. 2019;74:652-9.

29. Anstey DE, Muntner P, Bello NA, et al. Diagnosing Masked Hypertension Using Ambulatory Blood Pressure Monitoring, Home Blood Pressure Monitoring, or Both? Hypertension. 2018;7:1200-7.

30. Shimbo D, Abdalla M, Falzon L, Townsend RR, Muntner P. Studies comparing ambulatory blood pressure and home blood pressure on cardiovascular disease and mortality outcomes: a systematic review. J Am Soc Hypertens. 2016;10:224-234.e17.

31. Fagard RH, Van Den Broeke C, De Cort P. Prognostic significance of blood pressure measured in the office, at home and during ambulatory monitoring in older patients in general practice. J Hum Hypertens. 2005;19:801-7.

32. Pierdomenico SD, Lapenna D, Bucci A, Tommaso RD, Mascio RD, Manente BM, et al. Cardiovascular Outcome in Treated Hypertensive Patients with Responder, Masked, False Resistant, and True Resistant Hypertension. Am J Hypertens. 2005; 18:1422-8.

33. Ohkubo T, Kikuya M, Metoki H, et al. Prognosis of "Masked" Hypertension and "White-Coat" Hypertension Detected by 24-h Ambulatory Blood Pressure Monitoring: 10-Year Follow-Up From the Ohasama Study. J Am Coll Cardiol. 2005;46:508-15.

34. Ben-Dov IZ, Kark JD, Mekler J, Shaked E, Bursztyn M. The white coat phenomenon is benign in referred treated patients: a 14-year ambulatory blood pressure mortality study. J Hypertens. 2008;26:699-705.

35. Hansen T, Jeppesen J, Rasmussen S, Ibsen H, Torppedersen C. Ambulatory Blood Pressure Monitoring and Risk of Cardiovascular Disease: A Population Based Study. Am J Hypertens. 2006;19:243-50.
36. Mancia G, Facchetti R, Bombelli M, Grassi G, Sega R. Long-Term Risk of Mortality Associated With Selective and Combined Elevation in Office, Home, and Ambulatory Blood Pressure. Hypertension. 2006;47:846-53.

37. Pierdomenico SD, Pannarale G, Rabbia F, et al. Prognostic Relevance of Masked Hypertension in Subjects With Prehypertension. Am J Hypertens. 2008;21:879-83.

38. Kushiro T, Kario K, Saito I, et al. Increased cardiovascular risk of treated white coat and masked hypertension in patients with diabetes and chronic kidney disease: the HONEST Study. Hypertens Res. 2017;40:87-95.

39. Hermida RC, Ayala DE, Mojón A, Fernández JR. Sleep-Time Blood Pressure and the Prognostic Value of Isolated-Office and Masked Hypertension. Am J Hypertens. 2012;25:297-305.

40. Hänninen M-R, Niiranen T, Puukka P, Johansson J, Jula A. Prognostic significance of masked and white-coat hypertension in the general population: the Finn-Home Study. J Hypertens. 2012;30:705-12.

41. Minutolo R, Gabbai FB, Agarwal R, et al. Assessment of Achieved Clinic and Ambulatory Blood Pressure Recordings and Outcomes During Treatment in Hypertensive Patients With CKD: A Multicenter Prospective Cohort Study. Am J Kidney Dis. 2014;64:744-52.

42. Booth JN, Diaz KM, Seals S, et al. Masked Hypertension and Cardiovascular Disease Events in a Prospective Cohort of African Americans: the Jackson Heart Study. Hypertension. 2016; 68:501-10.

43. Pannarale G, Moroni C, Acconcia MC, et al. The natural history of prehypertension. A 20-year follow-up. Eur Rev Med Pharmacol Sci. 2017;21:1329-34.

44. Pierdomenico SD, Pierdomenico AM, Coccina F, Porreca E. Prognosis of Masked and White Coat Uncontrolled Hypertension Detected by Ambulatory Blood Pressure Monitoring in Elderly Treated Hypertensive Patients. Am J Hypertens. 2017;30:1106-11.

45. Tocci G, Presta V, Figliuzzi I, et al. Prevalence and clinical outcomes of whitecoat and masked hypertension: Analysis of a large ambulatory blood pressure database. J Clin Hypertens. 2018;20:297-305. 